\title{
The impact of lecture chunking format on university student vigilance: Implications for classroom pedagogy
}

\author{
Andrew Harris ${ }^{1}$, Sarah Buglass ${ }^{1}$ and Georgina Gous ${ }^{2}$ \\ ${ }^{1}$ NTU Pscychology, Nottingham Trent University, United Kingdom; 2University of Lincoln, School of Psychology, \\ United Kingdom
}

Correspondence should be addressed to Dr. Andrew Harris (iDａndrew.harris02@ntu.ac.uk Received 28 June 2021; Revised 17 September 2021; Accepted 10 October 2021

\begin{abstract}
Consistent with capacity theories of attention, attention can be sustained to the extent that spare mental resources remain available. The traditional lecture in higher education has received criticism for being too long to hold a student's attention. This is based on several author's claims that there is a measurable decrement in student attention after approximately 10-15 minutes of sustained content delivery. The present research aimed to investigate if providing small, separate units of an asynchronous lecture is able to enhance motivation for task engagement through perceived achievability of the learning outcomes, and consequently, enhance sustained attention amongst postgraduate university students. Utilising a quasiexperimental design, 51 postgraduate psychology students were recruited by opportunistic sampling from a cognitive psychology lecture on an MSc Psychology course, and given the option to watch either a long, single-video version of a lecture, or the same lecture delivered as smaller separate video chunks. Key findings indicate that presenting the material as smaller separate video units increased the perceived achievability of the learning outcomes and reduced the number of attention lapses experienced, but not the duration of those lapses, all measured via self-report single-item measures. The shorter separate videos condition also saw greater levels of break taking compliance. Looking at the sample as a whole using a hierarchical regression analysis, whilst controlling for student mind wandering tendencies as measured by the Mind Excessively Wandering Scale (MEWS), taking breaks was a significant negative predictor of attention lapses. Taken together, this suggests taking breaks is an integral part of sustained attention, and that chunking lectures into separate video units increases break taking compliance. Therefore, when designing online asynchronous learning material, lecturers should consider the value of chunking learning material for its potential direct and indirect effect on sustained attention.
\end{abstract}

Keywords: Asynchronous lectures, attention, capacity theory, chunking, vigilance

\section{Introduction}

In more recent years, the efficacy of the traditional lecture has come under scrutiny for its potential lack of effectiveness, in terms of its contribution to knowledge retention and student outcomes (Bradbury, 2016). Much of this discussion has been stimulated by a general paradigm shift in dominant learning theory, where recent decades have seen a transition from a more passive, behaviourist view of learning, to a more active, cognitivist constructivism approach (Evans \& Waring, 2011). The implications this has for higher education, which will be the focus of the present paper, is that more emphasis is placed on the interaction between structural and situational characteristics of the learning environment and human cognitive factors in the assimilation of information.

Vigilance, the ability to sustain concentrated attention over a prolonged period of time (Warm et al., 2008), is one such cognitive factor that interacts with lecture format to determine learning outcomes (Young et al., 2009). The traditional lecture, which typically lasts 50-60 minutes, has received criticism for being too long to hold a student's attention (Bradbury, 2016). This is based on several author's claims that there is a measurable decrement in attention after approximately 10-15 minutes of sustained content delivery. Whilst the claim of a 10-15 minute attention span in lectures 
appears ubiquitous in pedagogical texts (e.g., Davis, 1993; McKeachie, 1986; Wankat, 2002; for a review, see Bradbury, 2016), the empirical basis for this assumption is contentious.

The aforementioned papers here, make claims not based on primary research evidence, but all cite the same primary resource (i.e., Hartley \& Davies, 1978) as the origin of the 10-15 minute attention span assumption. A closer examination of Hartley and Davies (1978) reveals that the main focus of the research was notetaking and not attention per se. It was observed that the quantity of notes taken by students declined after 10-15 minutes. This has subsequently been assumed as a proxy measure for attention. However, Hartley (Hartley \& Cameron, 1967) concedes that notetaking is not a good proxy measure for attention, and that any decline in notetaking itself could result from a synchronisation with the amount and pertinence of the content being delivered during a lecture at any given time. Furthermore, using a direct observation method, Johnstone and Percival (1976) observed 90 lectures and noted that there was an attention lapse within the first five minutes of the class, and again within the 10-18 minute point of the lecture. However, there are several noteworthy limitations in this study, namely, $87 \%$ of the classes observed were done so by a single observer. The most obvious caveat however, was that there was no definition provided as to what constituted an attention lapse. This is particularly problematic when considering external observations may misinterpret a student looking away from the front of the class as an attentional lapse, when in fact, it could represent reflective thought and assimilation of ideas. Contrary, a student looking at the front of the class towards the lecturer may be experiencing mind wandering and not actually focusing on the lecture content.

Modern technology, with particular reference to the modern mobile phone, poses further competition for attentional resources in the classroom and represents a significant source of distraction. McCoy (2016) conducted a survey of American college-aged students and found that across the 675 participants, they reported an average of $21 \%$ of their time in class was spent on a digital device for non-class purposes. This competition for attentional resources emphasises the need to design and structure lectures in a manner capable of engaging the student and holding their attention.

More recent research utilising portable eye-tracking technology suggests the level of studentstudent and student-lecturer interaction plays a vital role in student on-task vigilance. Rosengrant et al. (2021) demonstrated that on-task vigilance percentages starting with $67 \%$ at the start of class rose to an average of above $90 \%$ on-task vigilance at the 7 to 9 -minute mark with minor fluctuation. The authors concluded that well-structured classes punctuated by interactions can be an effective method of maintaining student vigilance for entire class sessions, not just the first 10 minutes. However, these more contemporary methods of measuring student vigilance suffer from the same limitations as observation methods, in the sense that fixation on task-related material may not signify full attention is being paid to the material.

\subsection{Benefits of 'Chunking' Learning Material}

According to the attention-resource model (Davies \& Parasuraman, 1982; see also Warm et al., 2008 for a review), mental capacity is widely considered a finite resource. This suggests attention can be sustained to the extent that spare mental resources remain available. Once cognitive capacity has been reached, the ability to maintain attention and process new information is hindered, as evidenced in numerous studies within cognitive psychology and cognitive neuroscience (for a review, see Oberauer, 2019). Studies using self-report methods demonstrate an association between difficulties in sustaining attention and perceived increases in task workload (Warm et al., 1996). This evidence is corroborated with functional neuroimaging studies demonstrating that as task workload increases, there is a restriction in blood flow to areas of the brain associated with attentional control immediately before lapses in attention (Weissman et al., 2006). These brain areas, such as the frontal and parietal cortical regions, have been shown to be less active even after performing cognitively demanding tasks (Lim et al., 2010), highlighting the persistent and lasting effects of high workload on attentional processes. Experimental research has demonstrated that the use of alerting tones and warning cues can enhance vigilance (McLean et al., 
2009; O'Connor et al., 2011). However, more practical and less intrusive solutions are required to facilitate student's sustained attention during lectures.

A commonly employed strategy to combat this reported attention decrement within an education context is the use of 'chunking' and micro-learning (see e.g., Jahnke et al., 2020; Major \& Calandrino, 2018). Although conceptually similar, there are arguably some noteworthy differences between chunking and micro-learning. Micro-learning aims to deliver learning material in short, manageable, and attainable packages for the learner, designed with adult-learners needs in mind with respect to self-direction and pacing of learning. Micro-learning is often used as a prerequisite to, or means to supplement, more formal learning, or to prepare learners for more formal learning environments (Cole \& Torgerson, 2017). Whilst still a strategy for making learning more manageable for students, chunking is not intended as a prerequisite to more formal learning and does not necessarily require the re-writing and re-design of existing learning material. It is a simpler restructuring process, where existing material, within a lecture for example, is broken up into several smaller units.

Therefore, presenting information in lectures in smaller, more manageable chunks, may have the benefit of reducing cognitive load and facilitating sustained attention. Many lecturers will naturally divide their lectures into distinguishable but related subsections, often communicated at the start of the lecture in the form of a lecture 'scope'. However, as lectures chunked in this format still form a single piece of learning material and are delivered as a single entity i.e., one recorded lecture, it may still result in cognitive overload and make it challenging for students to sustain attention. The present paper argues that dividing a pre-recorded lecture into more distinguishable parts, by creating a series of smaller separate recorded units of the lecture, may facilitate student attention. It is argued that this could be effective and explained by an increase in motivation provided by the measurable progress effect (Rowe et al., 2017). From this perspective, it is argued that when goals such as absorbing the information in a lecture are viewed by the individual as achievable, this enhances motivation and is more likely to result in achieving the goal. By providing motivation for students by presenting material in smaller more distinguishable and manageable chunks, this in turn is likely to facilitate executive functions such as attention, where motivation has been demonstrated as being an important psychological variable capable of facilitating attention. For example, Neigel et al. (2017) demonstrated intrinsic motivation was correlated with vigilance and task engagement within a sample of young adults. Furthermore, Esterman et al. (2014) demonstrated that participants with high motivation, induced by external reward, had greater accuracy in a sustained attention task when compared to non-rewarded participants. However, both sets of participants had comparable sustained attention performance decrements over time, suggesting that both motivational lapses and a depletion of cognitive resources can influence sustained attention.

Therefore, the present research aimed to investigate if providing small, separate units of a lecture is able to enhance motivation for task engagement and consequently, enhance sustained attention amongst postgraduate university students. The research was conducted with the purpose of informing higher education institutions about the role of asynchronous lecture formatting in facilitating student engagement and vigilance. The research tested the following hypotheses:

(H1) Perceived achievability (PA) of the lecture learning outcomes will be a positive predictor of motivation to engage with the lecture content (ME).

(H2) PA and ME will be negative predictors of frequency of attention lapses.

(H3) PA and ME will be higher in the shorter videos condition compared to the one long video condition.

(H4) Frequency and duration of attentional lapses will be lower in the shorter videos condition compared to the one long video condition. 


\section{Method}

\subsection{Design}

A between-subjects quasi-experimental design was used to assess the impact of asynchronous lecture chunking format on student vigilance. A quasi- experimental design was chosen as it was necessary to allow students to self-select which lecture format they wished to view. The use of quasi-experimental designs in research of this nature is beneficial as it minimises threats to ecological validity through facilitation of a more naturalistic environment (DeRue et al., 2012). The independent variable was lecture chunking format, consisting of two conditions: one long video format; and multiple shorter videos format. The main dependent variables of interest were the frequency of attention lapses and the duration of attention lapses. Other dependent variables included self-report valence and arousal, as well as break compliance. All dependent variables were measured using a self-report questionnaire following the respective lecture.

\subsection{Participants}

Opportunity sampling was employed to acquire a sample of 51 postgraduate University students (of 83 enrolled) from a Cognitive Psychology module on an MSc Psychology course. Permission was granted from the module leader for obtaining participants to take part in the present study. The sample was a mix of full-time and second-year part-time students. This is considered a psychology conversion course, as students on this course do not have a BSc undergraduate degree in psychology or equivalent. It is typically a one-year course full-time, or two-year course if taken part-time.

\subsection{Materials}

\subsubsection{Lecture videos}

The two lecture formats were presented asynchronously on the cognitive psychology module on the MSc Psychology Course at NTU. The long video format was a single 80-minute recorded lecture media file. Within the video recording, the lecturer prompted students to pause the video and take a five-minute break three times: at the approximate (+/- 23 seconds) 20-minute; 40minute; and 60-minute time point. In the shorter videos condition, students were presented with the same lecture, but this was broken down into four 20-minute shorter videos. At the end of each short video, the lecturer encouraged students to take a five-minute break. The topic of the lecture was theories and models of attention, recorded by a 32-year old male lecturer from the psychology department. The vast majority of the recording was audio only, but the lecturer appeared on the video to introduce the lecture topic at the start of the lecture, again to prompt students to take breaks at the set times, and finally, at the end of the lecture to guide students where to post any follow-up lecture questions.

\subsubsection{Mind Excessively Wandering Scale (MEWS)}

Mind wandering tendencies were measured using the Mind Excessively Wandering Scale [MEWS] (Mowlem et al., 2019). The MEWS was initially developed as a 15-item scale, but previous psychometric evaluation and validation found 3 items had low factor loadings and that shortening the scale to 12-items did not reduce its sensitivity or specificity (Mowlem et al., 2019), so the 12item version was used in the present study. Example items include 'I can only focus my thoughts on one thing at a time with considerable effort', and 'I find my thoughts are distracting and prevent me from focusing on what I am doing'. Items are scored on a 4-point Likert-type scale ranging from 0- not at all or rarely; to 3-nearly all of the time or constantly. Internal consistency for the 12-item MEWS was high for the complete sample $(a=.89)$.

\subsubsection{Arousal and valence}

The original version of the Self-Assessment Manikins [SAMs] (Lang, 1980) were used to measure the participants' subjective levels of arousal and valence during the lecture they selected to watch. 
The SAMs are a non-verbal visual assessment technique designed to measure the subjective pleasure and arousal associated with affective responses to a wide variety of stimuli. The SAMs were chosen to assess valence and arousal as it has been demonstrated as a simple and efficient method for measuring affective responses to a range of stimuli or events, are ideal for remote delivery, and have been shown to be highly correlated with lengthier, verbal alternatives (Bradley \& Lang, 1994), such as the Semantic Differential Scale (see e.g., Russell \& Mehrabian, 1977). Fivepoint versions (1-5) of the SAMs were used in the present study.

\subsubsection{Vigilance}

To measure the frequency of attention lapses, students were asked one question created by the authors, 'During the lecture you have just watched, how many times did you notice a lapse in your attention? (E.g., mind wandering/day dreaming/thinking about something unrelated to the lecture)'. Responses were measured on a four-point Likert-scale and included the options: 1) not at all; 2) 1-2 times; 3) 3-4 times; 4) 5 or more times. To measure the duration of attention lapses, participants were asked a second question created by the authors, 'If you experienced any attention lapses during the lecture, please estimate the average duration of those lapses.' Responses were measured on a fourpoint Likert-scale and included the options: 1) a few seconds; 2) 30 seconds-1 minute; 3) 1-2 minutes; 4) more than 2 minutes.

\subsubsection{Break compliance}

To measure the rate of break compliance, participants were asked one question created by the authors, 'When prompted by the lecturer to do so, how often did you take a break?' Responses were measured on a four-point Likert-scale and included the options: 1) never; 2) some of the time; 3) most of the time; 4 ) always.

\subsubsection{Perceived achievability of learning outcomes and motivation to engage}

To measure the perceived achievability of the learning outcomes (PA), participants were asked 'To what extent do you agree with the following statement? 'By the end of the lecture, I was able to achieve all of the intended learning outcomes". Participants responses were recorded on a nine-point Likert-scale anchored at 1) completely disagree and 9) completely agree. Participants self-assessed levels of motivation to engage with the lecture content (ME) was measured with the question 'During the lecture, how motivated were you to engage with the lecture content?' Responses were recorded on a nine-point Likert-scale anchored at 1) no motivation and 9) my maximum level of motivation.

\subsection{Procedure}

The asynchronous lecture was a time-tabled event which students could access from 24 hours before the official time on the students' timetable. On the module learning page where the lecture could be downloaded, students were informed that they had a choice between two versions of the lecture: a single video version; or a multiple shorter videos version. They were told that other than this structural difference, the lecture was exactly the same in both versions and they should choose the format they wished to watch. At this stage, participants were not aware they were taking part in a psychology study. On the final slide of the lecture, students were invited to take part in the questionnaire phase of the study by following either an HTML link or QR code to the Qualtrics ${ }^{\circledR}$ survey. Alongside the questionnaire links, students were informed that participation in the questionnaire was entirely voluntary and did not form part of the lecture or module content. Before proceeding with the questionnaire, participants were informed what the study was about, their right to withdraw and right to not take part, and participants had to agree to the consenting information to proceed. The survey presented the questions to participants in the following order: 'Which version of the lecture did you just watch?' (Long video/multiple shorter videos); Valence ratings; Arousal ratings; Vigilance-based questions; PA; ME; break compliance; and finally, MEWS. Figure 1 provides a schematic illustration of the study procedure. 
Figure 1

Schematic Illustration of the Study Procedure

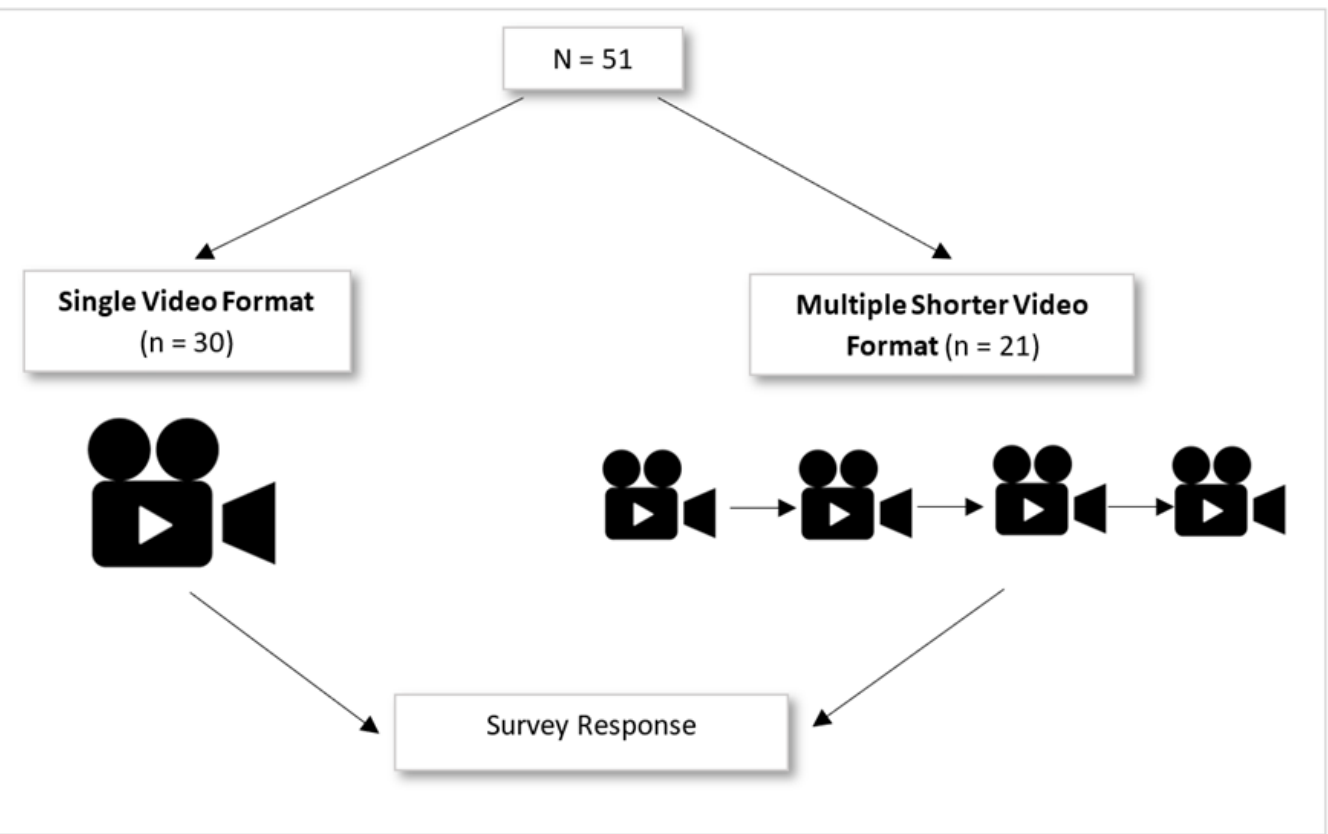

\subsection{Data analysis}

The data analyses were conducted using IBM ${ }^{\circledR}$ SPSS ${ }^{\circledR}$ Statistical Software (version 25). Betweensubjects t-tests were conducted to compare mean values for MEWS, valence, arousal, attention lapse frequency and duration, perceived achievability of the learning outcomes, motivation to engage, and break-taking compliance, across experimental conditions. The assumption of independence for t-test analysis was met, as the experimental groups were categorical in nature. There were no outliers identified in the data, using a 2 SD threshold. Assessment of the residuals and scatter plots confirmed normality, linearity, and equal variances were satisfied (Pallant, 2001). Hierarchical regression analysis was conducted to assess if frequency of attention lapses could be predicted by MEWS, PA, ME, and break compliance scores. The assumption of singularity was met, as the highest correlation value between the predictor variables was $r=.325, p=.02$ (PA and $\mathrm{ME}$ ). Assessment of the residuals and scatter plots confirmed normality, linearity, and equal variances were satisfied.

\section{Results}

Preliminary analysis on the data revealed that of the 51 participants in the overall sample, 30 had opted to watch the one long video version of the lecture, and 21 had opted to watch the shorter multiple videos version. Descriptive statistics for the data are provided in Table 1.

\section{Table 1}

Descriptive statistics for the short and long video conditions

\begin{tabular}{lcc}
\hline & Short Video Mean (SD) & Long Video Mean (SD) \\
\hline MEWS & $24.31(4.73)$ & $26.14(7.70)$ \\
Valence & $3.53(0.83)$ & $3.70(0.88)$ \\
Arousal & $3.07(1.03)$ & $3.09(1.16)$ \\
Attention lapse frequency & $2.19(0.81)$ & $2.77(0.82)$ \\
Attention lapse duration & $1.80(1.15)$ & $1.87(.87)$ \\
Perceived achievability & $6.19(1.36)$ & $5.40(1.10)$ \\
Motivation to engage & $6.24(1.45)$ & $6.00(1.26)$ \\
Break compliance & $3.24(1.09)$ & $2.50(1.14)$ \\
\hline
\end{tabular}




\subsection{Group-level Comparisons}

Between-subjects t-tests were used to compare the mean scores of the main study variables across the short video and long video lecture conditions. Despite higher scores being evident in the long video condition, comparisons with the short lecture condition rendered non-significant differences for mind wandering tendencies (MEWS), $t(49)=0.77, p=.45$, arousal, $t(49)=0.003, p=.96$, and valence, $t(49)=0.32, p=.57$. Furthermore, mean valence and arousal scores were above the midpoint of the five-point scales in both conditions, suggesting the lectures were overall enjoyed and that participants experienced moderate levels of autonomous arousal in both conditions.

Testing H4, a statistically significant difference was found between the mean scores for frequency of attention lapses, $t(49)=2.48, p=.017, d=0.71$, with more attention lapses in the long video condition than the shorter videos condition. This suggested a moderate effect size. Using data only from participants who reported experiencing at least one attention lapse, a further between subjects t-test showed that there was a non-significant difference between conditions in terms of the length of those attention lapses, $t(46)=0.05, p=.83$. Of note, only three of 51 participants reported having no attention lapses (two from the shorter videos condition, one from the longer video condition).

Comparisons between perceived achievability (PA) and motivation to engage (ME), testing H3, indicated that PA was statistically significantly higher in the shorter videos condition compared to the one long video condition, $t(49)=2.29, p=.027, d=0.64$, suggesting a moderate effect size. However, there were no statistically significant differences found for ME between the one long video and the shorter videos condition, $t(49)=0.63, p=.535$.

Finally, the rate at which participants complied with the lecturer's prompt to take a break (break compliance) rendered a statistically significant difference in the amount of breaks taken between the long video condition and the shorter videos condition, $t(49)=0.38, p=.025, d=0.87$, suggesting a large effect size. Participants in the short video condition were found to be more compliant to the prompts.

\subsection{Regression analysis}

To test $\mathrm{H} 1$ and $\mathrm{H} 2$ regression analyses were performed on data from the full sample.

\subsubsection{Perceived achievability (PA) as a predictor of motivation to engage (ME)}

The hypothesised relationship between PA and ME (H1) was tested using a simple linear regression. PA was a significant positive predictor of $\mathrm{ME}, F(1,49)=5.79, p=.02$, adjusted $\mathrm{R}^{2}=.087$. The regression coefficient, $B=0.34(\mathrm{SE}=0.14)$ indicated that a one-point increase in PA rating was associated with a 0.34 increase in ME rating.

\subsubsection{Predicting frequency of attention lapses from $M E W S, P A, M E$, and break compliance}

A three-stage hierarchical multiple regression (Table 2), with all relevant assumptions of the statistical analysis tested, was used to consider the relationship between the predictor variables MEWS, PA, ME and break compliance and the outcome variable, frequency of attention lapses (H2). Firstly, a sample size of 51 was deemed conservative, leading to the decision to use the adjusted $R^{2}$ value as an indicator of the variance in the outcome variable explained by the model.

Participant MEWS values were entered at stage one of the model to control for the participants' general mind wandering tendencies. PA and ME were entered at stage two, and break compliance at stage three. The hierarchical multiple regression revealed that at stage one, MEWS scores contributed significantly to the regression model, $F(1,49)=7.58, p=.008$, and accounted for $11.6 \%$ of the variance in frequency of attention lapses. The introduction of PA and ME at stage two explained an additional $0.5 \%$ of the variance in frequency of attention lapses, a statistically nonsignificant change (sig $F$ change $=.332$ ), but the overall model at stage two remained statistically 
Table 2

Hierarchical regression analysis with frequency of attention lapses as outcome

\begin{tabular}{lccccccc}
\hline \multirow{2}{*}{ Model } & \multirow{2}{*}{$B$} & \multicolumn{2}{c}{$95 \%$ CI } & \multirow{2}{*}{$\beta$} & $t$ & $p$ & $\begin{array}{c}\text { Adjusted } \\
R^{2}\end{array}$ \\
\cline { 3 - 4 } & & Step 1 & & & & & \\
MEWS & 0.06 & 0.02 & 0.10 & 0.37 & 2.75 & .008 & .12 \\
Step 2 & & & & & & & .12 \\
MEWS & 0.05 & 0.01 & 0.09 & 0.31 & 2.23 & .031 & \\
PA & -0.12 & -0.32 & 0.07 & -0.18 & -1.26 & .216 & \\
ME & -0.04 & -0.22 & 0.15 & -0.06 & -0.40 & .690 & \\
Step 3 & & & & & & & .22 \\
MEWS & 0.05 & 0.01 & 0.09 & 0.30 & 2.25 & .029 & \\
PA & -0.08 & -0.26 & 0.12 & -0.11 & -0.80 & .431 & \\
ME & -0.01 & -0.18 & 0.17 & -0.01 & -0.07 & .944 & \\
Break & -0.26 & -0.45 & -0.06 & -0.35 & 2.68 & .010 & \\
Compliance & & & & & & & \\
\hline
\end{tabular}

significant, $F(3,47)=3.29, p=.029$. Finally, at stage three, break compliance was added to the model, which explained an additional $10.2 \%$ of the variance in frequency of attention lapses, which was a statistically significant change (sig $F$ change $=.01$ ). At stage three, with all predictor variables added, the model accounted for $22.3 \%$ of the variance in frequency of attention lapses, which was a statistically significant model, $F(4,46)=4.59, p=.003$, but only MEWS values $(p=.029)$ and break compliance $(p=.01)$ were significant positive and negative predictors, respectively, of attention lapses.

\section{Discussion}

The research aimed to investigate if providing small, separate units of a lecture is able to enhance motivation for task engagement and consequently, enhance sustained attention amongst postgraduate university students. In support of hypothesis 1, data analysis of the sample as a whole showed that perceived achievability of the learning outcomes (PA) was a positive predictor of motivation to engage with the lecture content (ME). This is an important finding, particularly considering research that demonstrates increased motivation and engagement is associated with better learning outcomes, not only in higher education, but for all students (for a review see Panigrahi et al., 2018). However, a degree of caution is required when interpreting the direction of this relationship. Research has also demonstrated that higher achievers are more motivated (Gholami et al., 2012; Maccoby, 1995). Therefore, motivation and the associated enhanced capabilities of motivated individuals could enhance the perceived achievement of learning outcomes. As a result, the relationship between PA and ME might be best considered as bidirectional.

In partial support of hypothesis 3, the results of the present study showed that the manipulation of chunking format was associated with changes in PA, with higher achievability ratings recorded in the separate videos format. However, the manipulations of chunking format did not result in group-level differences in $\mathrm{ME}$, suggesting that any reduction in attention lapses resulting from chunking format was independent of a motivational effect. The finding that PA was greater and that attention lapses were fewer in the separate videos condition, but that there were no differences in ME between groups, suggests formatting recorded lectures into smaller but separate sections may make learning the content appear more achievable and reduce cognitive load, and therefore, reduce the number of attentional lapses experienced by students

Perhaps unsurprisingly, stage 1 of the hierarchical regression showed that MEWS scores were a significant positive predictor of frequency of attention lapses within the lecture. However, stage 2 of the regression analysis failed to provide support for hypothesis 2, as PA and ME were nonsignificant predictors of frequency of attention lapses when controlling for the variance accounted 
for by participant MEWS scores. Further analysis revealed that adding the number of breaks taken during the lecture significantly improved the regression model whilst controlling for MEWS, PA, and ME scores, where an increase in the number of breaks taken predicted fewer attention lapses. Analysis of the group-level data showed a statistically significant difference in the mean number of breaks taken when prompted between the lecture chunking conditions, with more breaks being taken in the shorter separate videos format. In corroboration with sample-level data analysis showing that the number of breaks taken was a negative predictor of frequency of attention lapses, this suggests the utilisation of breaks is an important factor in maintaining attention throughout a lecture. As the different lecture chunking formats did not result in group-level differences in motivation, the role of breaks in sustaining attention appears to be more in line with capacity theories of attention (see e.g., Warm et al., 2008) rather than any attentional benefits derived from enhanced motivation (for discussion, see Massar et al., 2016). Although there is much ongoing academic discussion on how taking breaks aids attention (e.g., Lee et al., 2015; Schmidt et al., 2016), one proposed theory suggests that taking breaks appears to aid attention by allowing a replenishment of cognitive resources (Tyler \& Burns, 2008), which is consistent with capacity theories of attention. Furthermore, research has demonstrated that taking longer breaks bias participants toward greater effort expenditure on task performance following the break, even when cognitive resources may not have been fully replenished (Lim \& Kwok, 2015).

Reasons for the finding that more breaks were taken when prompted in the shorter separate videos condition could include the fact that they are viewed as distinct elements of a lecture and therefore, more conducive to taking breaks between segments. Although the one long video version of the lecture had break prompts at the same location in the lecture as the separate videos condition, and was also divided into within-video chunks, having the material presented within one single recording more closely resembles live lectures where it is less common to have a break in one-hour lectures that involve for example, leaving the lecture theatre, walking around, going for a refreshment, etc. In this sense, having asynchronous lectures recorded as one single video stream more closely matches expectations and pre-conceived behavioural scripts about the format of lectures. In addition, the fact that in the separate videos condition participants would have to actively locate and load the next video segment creates a more natural break in the material and a shift in mental set. All participants would have to do in the long video version to not take a break would be to simply not click pause when prompted to take a break, meaning there is not as obvious a switch in mental set.

In partial support of hypothesis 4, frequency of attentional lapses was statistically significantly less in the shorter videos condition compared to the long video condition, but there were no group level differences in terms of duration of those attention lapses. This finding suggests that the way a lecture is chunked can affect student levels of attention. The data here suggests that this effect is independent of the students' motivation to engage, which was high in both conditions. This is perhaps due to the fact that the design of the study meant that students had a choice over the format of the lecture they watched. Consistent with self-determination theory (Deci \& Ryan, 2012), being given a choice and therefore, greater levels of control over one's learning, can enhance the learner's motivation to engage with the material. An alternative explanation for the generally high levels of motivation to engage across the sample could be accounted for by the fact this was a sample of postgraduate students, where motivation to engage with course content is frequently shown to be higher when compared to their undergraduate counterparts (Artino Jr \& Stephens, 2009).

\subsection{Implications}

Whilst the results here indicate that chunking asynchronous lectures into separate video units has benefits for perceived achievability of the learning outcomes and reduces attention lapses, generalisability of the findings to live online or face-to-face lectures is limited. There are some key factors that distinguish live lectures from asynchronous lectures, which includes the dynamic relationship between teacher and student. Not only can a teacher ad hoc adapt features such as the 
pitch and tone of their voice to emphasise key points and capture students' attention if they detect attention decrements, they can also interact with students in a much more dynamic sense compared to pre-recorded lectures. For example, a teacher can ask students a direct question in a live lecture, whereas questions posed in asynchronous lectures are more likely to be indirect 'pause and reflect' styles of questioning. Research shows that making lectures more dynamic with the inclusion of student participation in the form of answering questions has added benefits for attention levels (Bunce et al., 2010). Recent research has also demonstrated that the use of quizzes as a form of student participation, for example, Kahoot!, triggers positive attention and focus in the classroom (Licorish et al., 2018). Furthermore, the authors suggested that interacting with Kahoot! enabled students to take a break in the lecture and provided a point of difference.

One of the key findings from the present study was that chunking recorded lectures into separate video units encourages the uptake of breaks, which in turn, was a negative predictor of attention lapses. Given the limited estate space at brick and mortar universities, as well as limits to teaching staff time-based resources, it might not be practical for students to be taking frequent breaks given the large amount of information that needs to be presented in a limited amount of time, typically one hour. This therefore makes the recommendation of the use of regular breaks to aid attention fairly limited to asynchronous teaching events. Future research should continue to investigate ways of aiding student attention in live lectures without the need for regular breaks to replenish attention resources.

The preliminary analysis of students' affective response to the different lecture formats showed no statistically significant difference between groups. However, approximately $60 \%(30 / 51)$ of the students opted to watch the one long video version of the lecture when given the choice. This could perhaps be due to the status quo bias effect (see e.g., Kahneman et al., 1991), as attending and watching lectures is typically undertaken as a single event, rather than a series of smaller events. In this view, watching the single video version of the lecture more closely resembles what students come to expect of a lecture. Future research should investigate if given the choice between long video versions and shorter multiple video versions of a lecture over time, students would stick with their original preferences or migrate to alternative versions of the lecture, which would provide greater insight into the students' preferred method of learning from online asynchronous materials.

Whilst prima facie a reduction in attention lapses is a positive factor in the learning process, future research should assess how these structural changes to chunking relate to grade outcomes. Caution should be taken to ensure that such chunking strategies do not result in unintended and maladaptive outcomes. Whilst there is a large body of research demonstrating chunking aids various aspects of memory (see e.g., Ellis, 1996), providing asynchronous lecture content as distinct video units could result in limiting students' ability to link the taught concepts across parts of the lecture. Therefore, the design of asynchronous content in this format should ensure that the relationship between concepts that may appear in separate video units are not lost.

\section{Conclusion}

The present study investigated if providing small, separate units of a lecture is able to enhance motivation for task engagement and consequently, enhance sustained attention amongst postgraduate university students. It was found that chunking the lectures into smaller separate video units, compared to a single longer video, resulted in more breaks being taken, fewer attentional lapses, as well as higher ratings of perceived achievability of the learning outcomes. It found that whilst controlling for student mind wandering tendencies, the number of breaks taken during a lecture was a negative predictor of attention lapses during the lecture. The absence of motivation as a predictor of attention lapses suggest that breaks reduce attention lapses by reducing cognitive load. Future research should investigate how these structural changes to lecture chunking format, which show benefits for student attention, are associated with student academic performance. 


\section{References}

Artino Jr, A. R., \& Stephens, J. M. (2009). Academic motivation and self-regulation: A comparative analysis of undergraduate and graduate students learning online. The Internet and Higher Education, 12(3-4), 146-151. https:/ / doi.org/10.1016/j.iheduc.2009.02.001

Bradbury, N. A. (2016). Attention span during lectures: 8 seconds, 10 minutes, or more? Advances in Physiology Education, 40(4), 509-513. https:/ / doi.org/10.1152/advan.00109.2016

Bradley, M. M., \& Lang, P. J. (1994). Measuring emotion: the self-assessment manikin and the semantic differential. Journal of Behavior Therapy and Experimental Psychiatry, 25(1), 49-59. https:/ / doi.org/10.1016/0005-7916(94)90063-9

Bunce, D. M., Flens, E. A., \& Neiles, K. Y. (2010). How long can students pay attention in class? A study of student attention decline using clickers. Journal of Chemical Education, 87(12), 1438-1443. https:// doi.org/10.1021/ed100409p

Cole, M., \& Torgerson, C. (2017). Highlights from ATD's new micro-learning research report [Webinar]. http:/ / webcasts.td.org/ webinar/2266

Davies, D. R., \& Parasuraman, R. (1982). The Psychology of Vigilance. Academic Press.

Davis, B. G. (1993) Tools for Teaching. Jossey-Bass.

Deci, E. L., \& Ryan, R. M. (2012). Self-Determination Theory. In P. A. M. Van Lange, A. W. Kruglanski, \& E. T. Higgins (Eds.), Handbook of Theories of Social Psychology (p. 416-436). Sage Publications Ltd. http:/ / dx.doi.org/10.4135/9781446249215.n21

DeRue, D. S., Nahrgang, J. D., Hollenbeck, J. R., \& Workman, k. (2012). A quasi-experimental study of after-event reviewes and leadership development. Journal of Applied Psychology, 97(5), 997-1015. https:/ / doi.org/10.1037/a0028244

Ellis, N. C. (1996). Sequencing in SLA: Phonological memory, chunking, and points of order. Studies in Second Language Acquisition, 18, 91-126. https://www.jstor.org/stable/44487860

Esterman, M., Reagan, A., Liu, G., Turner, C., \& DeGutis, J. (2014). Reward reveals dissociable aspects of sustained attention. Journal of Experimental Psychology: General, 143(6), 2287-2296. https:// doi.org/10.1037/xge0000019

Evans, C., \& Waring, M. (2011). Student teacher assessment feedback preferences: The influence of cognitive styles and gender. Learning and Individual Differences, 21, 271-280. https:// doi.org/10.1016/j.lindif.2010.11.011

Gholami, R., Allahyar, N., \& Rafik-Galea, S. (2012). Integrative motivation as an essential determinant of achievement: A case of EFL high school students. World Applied Sciences Journal, 17(11), 1416-1424. http:/ / www.idosi.org/wasj/wasj17(11)12/6.pdf

Hartley, J. \& Cameron, A. (1967). Some observations on the efficiency of lecturing. Educational Review, 20(1), 30-37. https:/ / doi.org/10.1080/0013191670200103

Hartley, J. \& Davies, I.K. (1978). Note taking: A critical review. Programmed Learning and Educational Technology, 15(3), 207-224. https:/ / doi.org/10.1080/0033039780150305

Jahnke, I., Lee, Y. M., Pham, M., He, H., \& Austin, L. (2020). Unpacking the inherent design principles of mobile microlearning. Technology, Knowledge and Learning, 25(3), 585-619. https:/ / doi.org/10.1007/ s10758-019-09413-w

Johnstone, A. H., \& Percival, F. (1976). Attention breaks in lectures. Education in Chemistry, 13(2), 49-50. https:/ / doi.org/10.1080/00986280701291291

Kahneman, D., Knetsch, J. L., \& Thaler, R. H. (1991). Anomalies: The endowment effect, loss aversion, and status quo bias. Journal of Economic Perspectives, 5(1), 193-206. https:/ / doi.org/10.1257/jep.5.1.193

Lang, P. J. (1980). Self-Assessment Manikin. The Center for Research in Psychophysiology, University of Florida.

Lee, K. E., Williams, K. J., Sargent, L. D., Williams, N. S., \& Johnson, K. A. (2015). 40-second green roof views sustain attention: The role of micro-breaks in attention restoration. Journal of Environmental Psychology, 42, 182-189. https:/ / doi.org/10.1016/j.jenvp.2015.04.003

Licorish, S. A., Owen, H. E., Daniel, B., \& George, J. L. (2018). Students' perception of Kahoot!'s influence on teaching and learning. Research and Practice in Technology Enhanced Learning, 13(1), 1-23. https:/ / doi.org/10.1186/s41039-018-0078-8 
Lim, J., \& Kwok, K. (2016). The effects of varying break length on attention and time on task. Human Factors, 58(3), 472-481. https:/ / doi.org/10.1177/0018720815617395

Lim, J., Wu, W. C., Wang, J., Detre, J. A., Dinges, D. F., \& Rao, H. (2010). Imaging brain fatigue from sustained mental workload: an ASL perfusion study of the time-on-task effect. Neuroimage, 49(4), 3426-3435. https:/ / doi.org/10.1016/j.neuroimage.2009.11.020

Maccoby, M. (1995). Why Work: Motivating and Leading the New Generation (2nd ed.). Simon \& Schuster.

MacLean, K. A., Aichele, S. R., Bridwell, D. A., Mangun, G. R., Wojciulik, E., \& Saron, C. D. (2009). Interactions between endogenous and exogenous attention during vigilance. Attention, Perception, $\mathcal{E}$ Psychophysics, 71(5), 1042-1058. https:/ / doi.org/10.3758/APP.71.5.1042

Major, A., \& Calandrino, T. (2018). Beyond chunking: Micro-learning secrets for effective online design. FDLA Journal, 3(1), 1-5. https:/ / nsuworks.nova.edu/fdla-journal/vol3/iss1/13

Massar, S. A. A., Lim, J., Sasmita, K., \& Chee, M. W. L. (2016). Rewards boost sustained attention through higher effort: A value-based decision-making approach. Biological Psychology, 120, 2127. https://doi.org/10.1016/j.biopsycho.2016.07.019

McCoy, B. R. (2016). Digital distractions in the classroom phase II: Student classroom use of digital devices for non-class related purposes. Journal of Media Education, 7(1), 5-32. http:/ / digitalcommons.unl.edu/journalismfacpub/90

McKeachie, W. J. (1986). Teaching Tips: Strategies, Research and Theory for College and University Teachers. Lexington, MA: Heath. https:/ / doi.org/10.2307/328598

Mowlem, F. D., Agnew-Blais, J., Pingault, J. B., \& Asherson, P. (2019). Evaluating a scale of excessive mind wandering among males and females with and without attention-deficit/hyperactivity disorder from a population sample. Scientific Reports, 9(1), 1-9. https:/ / doi.org/10.1038/ s41598-01939227-W

Neigel, A. R., Claypoole, V. L., Waldorf, K. M., Dever, D. A., \& Szalma, J. L. (2017, September). Motivational correlates of vigilance task engagement. In Proceedings of the Human Factors and Ergonomics Society Annual Meeting (Vol. 61, No. 1, pp. 1524-1528). SAGE Publications. https:/ / doi.org/10.1177/1541931213601865

Oberauer, K. (2019). Working memory and attention-A conceptual analysis and review. Journal of Cognition, 2(1), 1-23. http:// doi.org/10.5334/joc.58

O'Connor, C., Robertson, I. H., \& Levine, B. (2011). The prosthetics of vigilant attention: Random cuing $\begin{array}{llll}\text { cuts } \quad \text { processing 25(4), 535-543. } & \text { demands. Neuropsychology, }\end{array}$ https:/ / doi.org/10.1037/a0022767

Pallant, J. (2001). SPSS Survival Manual. Allen \& Unwin.

Panigrahi, R., Srivastava, P. R., \& Sharma, D. (2018). Online learning: Adoption, continuance, and learning outcome-A review of literature. International Journal of Information Management, 43, 1-14. https:/ / doi.org/10.1016/j.ijinfongt.2018.05.005

Rosengrant, D., Hearrington, D., \& O'Brien, J. (2021). Investigating student sustained attention in a guided inquiry lecture course using an eye tracker. Educational Psychology Review, 33, 11-26. https:/ / doi.org/10.1007/s10648/020/09540/2

Rowe, D. A., Mazzotti, V. L., Ingram, A., \& Lee, S. (2017). Effects of goal-setting instruction on academic engagement for students at risk. Career Development and Transition for Exceptional Individuals, 40(1), 25-35. https:/ / doi.org/10.1177/2165143416678175

Russell, J. A., \& Mehrabian, A. (1977). Evidence for a three-factor theory of emotions. Journal of Research in Personality, 11(3), 273-294. https:/ / doi.org/10.1016/0092-6566(77)90037-X

Schmidt, M., Benzing, V., \& Kamer, M. (2016). Classroom-based physical activity breaks and children's attention: cognitive engagement works! Frontiers in Psychology, 7, 1474. https:/ / doi.org/10.3389/fpsyg.2016.01474

Tyler, J. M., \& Burns, K. C. (2008). After depletion: The replenishment of the self's regulatory resources. Self and Identity, 7(3), 305-321. https:/ / doi.org/10.1080/15298860701799997

Wankat, P.C. (2002). The Effective Efficient Professor: Teaching, Scholarship and Service. Allyn and Bacon. https:// doi.org/10.1080/1937156X.2003.11949512

Warm, J. S., Dember, W. N., \& Hancock, P.A. (1996). Vigilance and workload in automated systems. In R. Parasuraman, \& M. Mouloua (Eds.), Automation and human performance: Theory and applications (pp. 183-200). Erlbaum. 
Warm, J. S., Parasuraman, R., \& Matthews, G. (2008). Vigilance requires hard mental work and is stressful. Human Factors, 50(3), 433-441. https:/ / doi.org/10.1518/001872008X312152

Weissman, D. H., Roberts, K. C., Visscher, K. M., \& Woldorff, M. G. (2006). The neural bases of momentary lapses in attention. Nature Neuroscience, 9, 971-978. https:/ / doi.org/10.1038/nn1727

Young, M. S., Robinson, S., \& Alberts, P. (2009). Students pay attention! Combating the vigilance decrement to improve learning during lectures. Active Learning in Higher Education, 10(1), 41-55. https:/ / doi.org/10.1177/1469787408100194 